\title{
Association Between the Seated Single-Arm Shot-Put Test With Isokinetic Pushing Force
}

\author{
Bryan L. Riemann and George J. Davies
}

\begin{abstract}
Context: Previous investigations have examined the reliability, normalization, and underlying projection mechanics of the seated single-arm shot-put (SSASP) test. Although the test is believed to reflect test limb strength, there have been no assessments determining whether test performance is directly associated with upper-extremity strength. Objective: To determine the relationship between isokinetic pushing force and SSASP performance and conduct a method comparison analysis of limb symmetry indices between the 2 tests. Design: Controlled laboratory study. Setting: Biomechanics laboratory. Patients (or Other Participants): Twenty-four healthy and physically active men $(\mathrm{n}=12)$ and women $(\mathrm{n}=12)$. Intervention $(\boldsymbol{s})$ : Participants completed the SSASP and isokinetic pushing tests using their dominant and nondominant arms. Main Outcome Measures: SSASP distance and isokinetic peak force. Results: Significant moderate to strong relationships were revealed between the SSASP distances and isokinetic peak forces for both limbs. The Bland-Altman analysis results demonstrated significantly $(P<.002)$ greater limb symmetry indices for the SSASP (both medicine balls) than the isokinetic ratios, with biases ranging from -0.094 to -0.159 . The limits of agreement results yielded intervals ranging from \pm 0.241 to \pm 0.340 and \pm 0.202 to \pm 0.221 from the biases. Conclusions: These results support the notion that the SSASP test reflects upper-extremity strength. The incongruency of the limb symmetry indices between the 2 tests is likely reflective of the differences in the movement patterns and coordination requirements of the 2 tests.
\end{abstract}

Keywords: dynamometry, functional performance, shoulder, evaluation

Compared with the lower-extremity, fewer functional performance tests are available for upper-extremity (UE) testing, along with a scarcity of data validating the few UE functional performance tests described. The seated single-arm shot-put (SSASP) test is a functional performance test, which replicates activities that require the UE to produce a short burst of maximal pushing activity. ${ }^{1}$ The test involves the participant "putting" a medicine ball for distance while in a long seated position against a wall. Investigations have examined the SSASP measurement properties, including reliability, ${ }^{2}$ normalization methods, ${ }^{3}$ and underlying projection mechanics. ${ }^{1}$ Although the test is believed to reflect test limb strength, ${ }^{1}$ there have been no assessments determining whether test performance is directly associated with UE strength.

Isokinetics is the gold standard for assessing muscular strength in orthopedic patient populations. ${ }^{4}$ While most isokinetic testing involves an isolated open kinetic chain joint test, a reliable UE push-pull isokinetic method also exists. ${ }^{5}$ Given the relative movement pattern similarity between the push-pull test with the SSASP, and because isokinetic dynamometry provides objective strength measures, the purpose of this investigation was to determine the relationship between concentric isokinetic pushing force and SSASP performance. A secondary purpose was to conduct a method comparison analysis of limb symmetry indices (LSIs; dominant to nondominant limbs) between the SSASP and isokinetic pushing force tests. Finally, a variety of medicine ball masses have been previously used ${ }^{1-3}$ without any evidence to support which ball mass may be ideal. Based on the ball mass recommendations from the study of the underlying SSASP projection mechanics, ${ }^{1}$ an additional purpose was to compare the relationships

The authors are with Biodynamics and Human Performance Center, Georgia Southern University-Armstrong Campus, Savannah, GA. Riemann (briemann@ georgiasouthern.edu) is corresponding author. using the 1- and 2-kg medicine balls. We hypothesized that there would be a strong direct relationship between isokinetic pushing force and SSASP performance, a strong agreement of LSI between the 2 testing methods, and that the relationships with the lighter ball would correlate stronger with the faster velocity isokinetic testing, while the heavier ball would correlate stronger with the slower velocity isokinetic testing.

\section{Methods}

A total of 24 physically active men $(\mathrm{n}=12)$ and women $(\mathrm{n}=12)$, volunteered for participation (23.7 [1.9] y, 1.78 [0.12] m, and 72.7 [16.9] $\mathrm{kg}$ ). Study eligibility included individuals between 18 and 30 years and being physically active for a minimum of 3 days per week (30 min/session), as per the American College of Sports Medicine guidelines. ${ }^{6}$ Participants were excluded if they selfreported any trunk or UE injuries requiring surgery or medical attention within the last year. The study attained Armstrong State University institutional review board approval, and before participation, all subjects completed a basic health questionnaire and signed an institutional review board approved informed consent form. Considering $\rho=.55$ to be clinically relevant, an a priori power analysis with $\alpha=.05$ and $\beta=0.2$ indicated that 23 participations were needed.

Participants underwent dominant and nondominant limb SSASP and isokinetic testing during a single 45-minute session. The order of limbs and testing, including ball masses $(0.97 \mathrm{~kg}$ and $2.27 \mathrm{~kg}$ ) and isokinetic velocities, were randomized between participants. Prior to completing testing, participants completed a 5-minute upper-body ergometer (Cybex Aerobic Ergometer, Boston, MA) bout at a pace corresponding to a rating of perceived exertion (modified Borg scale) between 10 and 12. Then, participants completed 30 seconds of arm warm-ups consisting of forwards, backs, and horizontal adduction-abduction swings. 
The SSASP technique duplicated procedures previously described. ${ }^{1,3}$ Following a progressive gradient warm-up of 4 trials, ${ }^{1,3}$ each limb completed 3 test trials using both medicine balls ( 6 total trials for each limb), and distances were measured. A 2-minute rest was given after the gradient warm-up and between limb-and-load combinations, ${ }^{1,2}$ while a 30 -second rest was given between trials. ${ }^{3}$ The average of 3 test trials for each limb was used for statistical analysis.

Concentric push pattern isokinetic testing at $0.24,0.43$, and $0.61 \mathrm{~m} / \mathrm{s}$ was conducted duplicating the methods described previously, 5 including a progressive gradient warm-up of 4 trials at each velocity, using the UE closed kinetic chain attachment on a stationary isokinetic dynamometer (Biodex Medical Systems, Inc, Shirley, NY). Participants were given the following instruction: "push and pull the hand grip throughout the full range of motion for 5 repetitions as hard and fast as you can." A 30-second rest was given between each velocity. The peak force registered within the set of 5 repetitions at each testing velocity was recorded for data analysis.

Following normality exploratory analysis, scatterplots between the SSASP distances and isokinetic pushing peak forces were created. As all relationships demonstrated linearity, Pearson correlational statistics were computed. LSIs were computed for both the SSASP distances and isokinetic push forces by dividing the dominant limb by the nondominant limb. To determine LSI method compatibility, a Bland-Altman ${ }^{7}$ analysis was conducted to determine bias and $95 \%$ limits of agreement (LOA). Prior to LOA computation, LSI difference normality and heteroscedasticity were evaluated. Statistical significance was considered at $\alpha=.05$.

\section{Results}

Descriptive statistics are presented in Table 1. Significant moderate to strong relationships were revealed between the SSASP distances and isokinetic peak forces for both medicine balls and limbs
(Table 2). The Bland-Altman analysis (Figure 1) results demonstrated significantly $(P<.002)$ greater LSI for the SSASP (both medicine balls) than the isokinetic ratios, with biases ranging from -0.094 to -0.159 (Table 3 ). The LOA results yielded intervals ranging from \pm 0.241 to \pm 0.340 and \pm 0.202 to \pm 0.221 from the biases for the 0.91 - and $2.27-\mathrm{kg}$ medicine balls, respectively.

\section{Discussion}

Based on previous evidence, ${ }^{1-3}$ this study's main purpose was to determine associations between shot-put distance and isokinetic pushing force production, with the goal of determining if the SSASP test sufficiently reflects muscle performance in circumstances where isokinetic testing is unavailable. By addressing this purpose, as well as considering the LSI computed from the 2 tests, we were also hopeful that the results would indicate which medicine ball mass was most compatible with isokinetic force production. Moderate to strong relationships were revealed for both medicine ball masses, thus supporting the previous notion that the SSASP test reflects UE strength ${ }^{1}$; however, because similar associations existed between the 2 ball masses, we cannot advocate for a particular medicine ball mass. Thus, clinicians can use either mass medicine ball with the SSASP test to reflect UE strength.

Despite having moderate to strong relationships between the tests across both limbs, the LSI method comparison analysis results did not yield test compatibility. While the significant systematic biases can be overcome with score adjustments, the wide LOA intervals, which indicate the magnitude of expected differences between the SSASP and isokinetically derived LSI ratios, preclude interpreting SSASP distances limb asymmetry as being solely indicative of underlying isokinetically determined strength differences. In retrospect, there are differences between the 2 tests that can possibly explain the LSI discrepancies. First, while both tests involved a pushing movement pattern, the precise UE motion

\section{Table 1 Descriptive Statistics (Mean [SD]) for the Seated Single-Arm Shot-Put Distances, Isokinetic Pushing Forces, and Limb Symmetry Indices}

\begin{tabular}{|c|c|c|c|c|c|}
\hline & \multicolumn{2}{|c|}{$\begin{array}{c}\text { Seated single-arm shot-put } \\
\text { distances, m }\end{array}$} & \multicolumn{3}{|c|}{ Isokinetic pushing forces, N/kg } \\
\hline & $0.97 \mathrm{~kg}$ & $2.27 \mathrm{~kg}$ & $0.61 \mathrm{~m} / \mathrm{s}$ & $0.43 \mathrm{~m} / \mathrm{s}$ & $0.24 \mathrm{~m} / \mathrm{s}$ \\
\hline Dominant limb & $5.26(1.41)$ & $3.81(1.06)$ & $4.61(1.76)$ & $5.11(2.04)$ & $5.88(2.18)$ \\
\hline Nondominant limb & $4.68(1.33)$ & $3.46(0.91)$ & $4.71(1.78)$ & $5.23(1.90)$ & $5.88(2.20)$ \\
\hline Limb symmetry indices & $1.13(0.10)$ & $1.10(0.07)$ & $1.00(0.18)$ & $0.97(0.10)$ & $1.01(0.10)$ \\
\hline
\end{tabular}

Table 2 Pearson Correlational Coefficients Between Isokinetic Pushing Force and Seated Single-Arm Shot-Put Distances

\begin{tabular}{llcc}
\hline & & \multicolumn{2}{c}{ Seated single-arm shot-put distances } \\
\cline { 3 - 4 } Isokinetic linear velocity & Limb & $\mathbf{0 . 9 1} \mathbf{~ k g}$ & $\mathbf{2 . 2 7} \mathbf{~ k g}$ \\
\hline $0.61 \mathrm{~m} / \mathrm{s}$ & Dominant & .807 & .791 \\
& Nondominant & .868 & .826 \\
$0.43 \mathrm{~m} / \mathrm{s}$ & Dominant & .776 & .755 \\
& Nondominant & .821 & .769 \\
$0.24 \mathrm{~m} / \mathrm{s}$ & Dominant & .790 & .797 \\
& Nondominant & .849 & .802 \\
\hline
\end{tabular}

Note: All coefficients were statistically significant $(P<.001)$. 

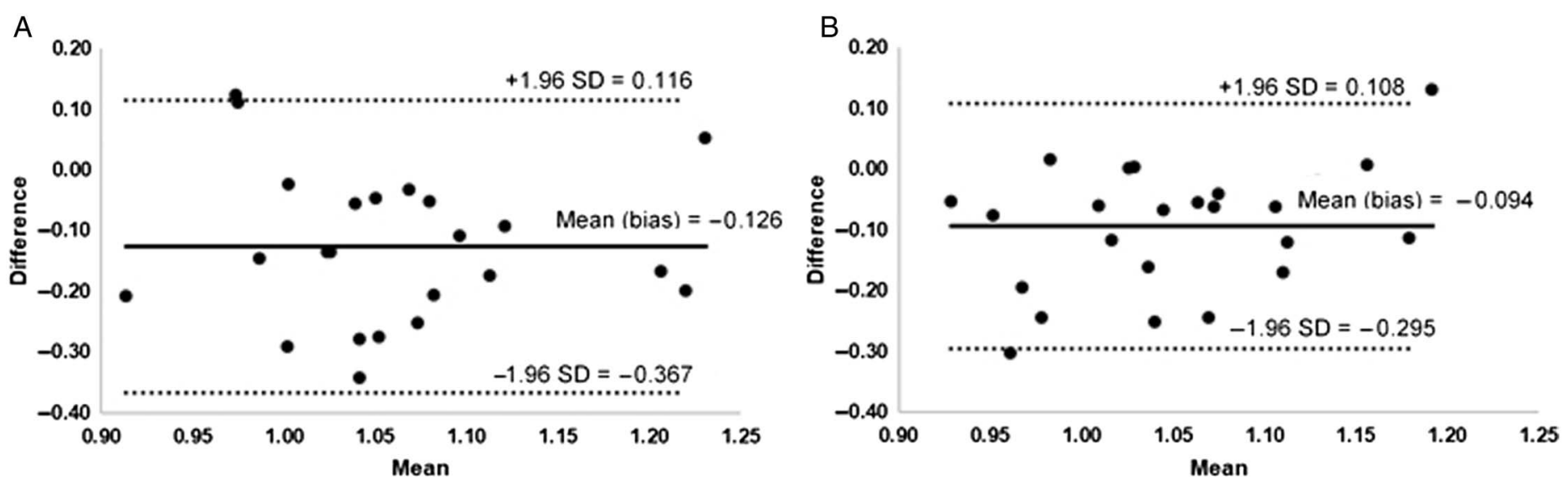

Figure 1 - Example Bland-Altman plots comparing the seated single-arm shot-put and isokinetic pushing limb symmetry indices. (A) 0.91-kg medicine ball and isokinetic pushing at $0.24 \mathrm{~m} / \mathrm{s}$. (B) $2.27-\mathrm{kg}$ medicine ball and isokinetic pushing at $0.24 \mathrm{~m} / \mathrm{s}$.

Table 3 Results of the Bland-Altman Analysis for the Limb Symmetry Indices

\begin{tabular}{|c|c|c|c|c|c|c|c|}
\hline \multirow{3}{*}{$\begin{array}{l}\text { Ball } \\
\text { mass }\end{array}$} & \multirow{3}{*}{$\begin{array}{c}\text { Isokinetic } \\
\text { velocity }\end{array}$} & \multicolumn{4}{|c|}{ Systematic bias } & \multicolumn{2}{|c|}{$\begin{array}{l}95 \% \text { limits of } \\
\text { agreement }\end{array}$} \\
\hline & & \multirow{2}{*}{$\begin{array}{c}\bar{X}_{\text {Diff }} \\
\left(S_{\text {Diff }}\right)\end{array}$} & \multirow{2}{*}{$\begin{array}{c}\text { Bias } \\
P \text { value }\end{array}$} & \multicolumn{2}{|c|}{$95 \% \mathrm{Cl}$ bias } & \multirow{2}{*}{$\begin{array}{l}\text { Lower } \\
\text { bound }\end{array}$} & \multirow{2}{*}{$\begin{array}{l}\text { Upper } \\
\text { bound }\end{array}$} \\
\hline & & & & Lower bound & Upper bound & & \\
\hline \multirow[t]{3}{*}{$0.91 \mathrm{~kg}$} & $0.61 \mathrm{~m} / \mathrm{s}$ & $-0.127(0.173)$ & .002 & -0.200 & -0.053 & -0.466 & 0.213 \\
\hline & $0.43 \mathrm{~m} / \mathrm{s}$ & $-0.159(0.148)$ & $<.001$ & -0.222 & -0.097 & -0.449 & 0.130 \\
\hline & $0.24 \mathrm{~m} / \mathrm{s}$ & $-0.126(0.123)$ & $<.001$ & -0.178 & -0.074 & -0.367 & 0.116 \\
\hline \multirow[t]{3}{*}{$2.27 \mathrm{~kg}$} & $0.61 \mathrm{~m} / \mathrm{s}$ & $-0.118(0.113)$ & $<.001$ & -0.167 & -0.069 & -0.339 & 0.103 \\
\hline & $0.43 \mathrm{~m} / \mathrm{s}$ & $-0.127(0.116)$ & $<.001$ & -0.354 & -0.078 & -0.354 & 0.100 \\
\hline & $0.24 \mathrm{~m} / \mathrm{s}$ & $-0.094(0.103)$ & $<.001$ & -0.137 & -0.050 & -0.295 & 0.108 \\
\hline
\end{tabular}

Abbreviations: CI, confidence interval; $\bar{X}_{\text {Diff }}$, mean difference; $\mathrm{SD}_{\text {Diff }}, \mathrm{SD}$ of differences. Note: Negative values indicate larger limb symmetry indices for the seated singlearm shot-put distances than the isokinetic push forces.

trajectory differed between the tests. The isokinetic pushing test comprises horizontal hand movement, whereas the terminal SSASP part involves the hand moving approximately $48^{\circ}$ above the horizontal. ${ }^{1}$ Consequentially, different muscles and muscle portions are likely active between the 2 tests. Supporting this explanation are the results of a study ${ }^{8}$ comparing flat and incline dumbbell bench presses, as the thorax to trajectory angles are compatible to this study. Although the bench press study only considered electromyography for several scapulothoracic and glenohumeral joint muscles, and did not consider elbow and wrist joint muscles, the concentric results documented very distinct muscle activation differences. ${ }^{8}$ Specifically, while the flat dumbbell press involved similar levels of muscle activation, the incline dumbbell press promoted significantly greater upper trapezius, anterior deltoid, and pectoralis major clavicular electromyography. ${ }^{8}$ By extension, while both tests in this study involve pushing movements, the SSASP may particularly require more muscular involvement for upward scapular rotation (eg, serratus anterior) and anterior deltoid/ pectoralis major clavicular to move the glenohumeral joint through a greater range of shoulder flexion than the isokinetic pushing test. Thus, while movement patterns between the 2 tests in this study were sufficiently similar to correlate, the subtleties in specific muscles active during each test might explain the lack of precision between the LSI method comparison analysis. Finally, for the SSASP, we used the average of 3 trials, whereas for the isokinetics, we used the peak force occurring across the 5 repetitions at each velocity. While the rationale for using the SSASP average and isokinetic peak forces were based on previous literature, ${ }^{1-3,5}$ the difference may have influenced the LSI computations and represents something for future research to consider.

A second potential explanation for the LSI comparison discrepancy could be the distal segment movement freedom. During the isokinetic pushing test, the handle/hand was constrained to follow a dictated linear path. In contrast, during the SSASP test, the movement of the hand through space was only constrained by the inertial properties of the medicine ball. Interestingly, it appears that the inertial medicine ball properties do not significantly influence terminal release angles nor heights, ${ }^{1}$ possibly explaining the association similarities between the 2 tests in this study across the 2 ball masses. Nonetheless, despite small and statistically insignificant differences between the dominant and nondominant limbs with regard to underlying SSASP projection mechanics, ${ }^{1}$ there are likely subtle coordination differences between the limbs that influence the shot-put distances thereby promoting larger and more variable LSI than the isokinetically derived LSI.

The LSI based on SSASP distances in this study were $113 \%$ and $110 \%$ for the 0.97 - and $2.27-\mathrm{kg}$ medicine balls, respectively. These values are very close to 3 previous studies investigating the SSASP test in healthy individuals, ${ }^{1-3}$ thereby further solidifying how clinicians can interpret a patient's test result. Furthermore, the 
shot-put distances are like those reported by the 2 previous investigations ${ }^{1,3}$ performing the test with a similar floor-based position.

In conclusion, the current results demonstrating SSASP associations with pushing force extend the previous research examining SSASP measurement-associated factors. ${ }^{1-3}$ Similar to the previous studies, ${ }^{1-3}$ this investigation only considered healthy young adults. Based on the documented measurement properties, LSI similarity across studies, and associations between SSASP distances with isokinetic pushing forces, the next step of SSASP research should consider evaluating patients. Specifically, we advocate examining whether the SSASP is sensitive to unilateral UE patients, as well as its association with patient-reported outcomes.

\section{References}

1. Riemann BL, Johnson W, Murphy T, Davies GJ. A bilateral comparison of the underlying mechanics contributing to the seated single-arm shot-put functional performance test. J Athl Train. 2018;53(10): 976-982. PubMed ID: 30299159 doi:10.4085/1062-6050-388-17

2. Negrete RJ, Hanney WJ, Kolber MJ, et al. Reliability, minimal detectable change, and normative values for tests of upper extremity function and power. J Strength Cond Res. 2010;24(12):3318-3325. PubMed ID: 21088548 doi:10.1519/JSC.0b013e3181e7259c
3. Chmielewski TL, Martin C, Lentz TA, et al. Normalization considerations for using the unilateral seated shot put test in rehabilitation. J Orthop Sports Phys Ther. 2014;44(7):518-524. PubMed ID: 24816499 doi:10.2519/jospt.2014.5004

4. Davies GJ, Riemann BL, Ellenbecker TS. Role of isokinetic testing and training after ACL injury and reconstruction: causes, impacts and conditioning programs. In: Noyes FR, Barber-Westin S, eds. ACL Injuries in the Female Athlete. 2nd ed. Berlin, Germany: SpringerVerlag; 2018:567-588.

5. Riemann BL, Davis SE, Huet K, Davies GJ. Intersession reliability of upper extremity isokinetic push-pull testing. Int J Sports Phys Ther. 2016;11(1):85-93. PubMed ID: 26900503

6. Garber C, Blissmer B, Deschenes M, et al. Quantity and quality of exercise for developing and maintaining cardiorespiratory, musculoskeletal, and neuromotor fitness in apparently healthy adults: guidance for prescribing exercise. Med Sci Sports Exerc. 2011;43(7):1334-1359. PubMed ID: 21694556 doi:10.1249/MSS. 0b013e318213fefb

7. Bland JM, Altman DG. Measuring agreement in method comparison studies. Stat Methods Med Res. 1999;8(2):135-160. PubMed ID: 10501650 doi:10.1177/096228029900800204

8. Luczak J, Bosak A, Riemann BL. Shoulder muscle activation of novice and resistance trained women during variations of dumbbell press exercises. J Sports Med. 2013;2013:1-6. PubMed ID: 26464884 doi:10.1155/2013/612650 